STUDI

FRANCESI

\title{
Studi Francesi
}

Rivista quadrimestrale fondata da Franco Simone

184 (LXII | I) | 2018

Varia

\section{MAURICE SCÈVE, Microcosme}

\section{Michele Mastroianni}

\section{OpenEdition \\ Journals}

Édition électronique

URL : https://journals.openedition.org/studifrancesi/11699

DOI : 10.4000/studifrancesi. 11699

ISSN : 2421-5856

\section{Éditeur}

Rosenberg \& Sellier

\section{Édition imprimée}

Date de publication : 1 avril 2018

Pagination : 120-121

ISSN : 0039-2944

\section{Référence électronique}

Michele Mastroianni, « MAURICE scève, Microcosme », Studi Francesi [En ligne], 184 (LXII | I) | 2018, mis en ligne le 02 juillet 2018, consulté le 15 novembre 2021. URL : http://journals.openedition.org/ studifrancesi/11699; DOI : https://doi.org/10.4000/studifrancesi.11699

Ce document a été généré automatiquement le 15 novembre 2021.

\section{(c) (†)}

Studi Francesi è distribuita con Licenza Creative Commons Attribuzione - Non commerciale - Non opere derivate 4.0 Internazionale. 


\title{
MAURICE SCÈVE, Microcosme
}

\author{
Michele Mastroianni
}

\section{RÉFÉRENCE}

MAURICE SCÈVE, Microcosme, édition de Michèle Clément, Paris, Classiques Garnier, 2016, «Classiques Jaunes», 390 pp.

1 Spécialiste de la Renaissance et du Baroque, connue en particulier pour ses études sur la poésie religieuse (Une poétique de crise: poètes baroques et mystiques (1570-1660), Paris, Champion, 1996), et ses essais sur le cynisme (Le Cynisme à la Renaissance: d'Érasme à Montaigne, suivi de "Les Espitres de Diogenes" (1546), Genève, Droz, 2005), Michèle Clément a codirigé, récemment, avec Janine Incardona, le volume L'émergence littéraire des femmes à Lyon à la Renaissance (1520-1560), Saint Étienne, Publications de l'Université de Saint-Étienne, 2008 et dirigé des travaux sur Étienne Dolet (1509-2009), Genève, Droz, 2012. Restant sur la poésie de la Renaissance, Michèle Clément publie maintenant cette excellente édition critique qui n'a pas seulement le mérite de fournir un instrument précieux à la fois au niveau philologique et au niveau de la reconstruction des sources classiques et modernes agissant sur Maurice Scève, mais aussi de présenter un travail très précis qui offre une importante introduction, au point qu'on pourrait la définir comme une étude monographique axée sur une dense digression, par laquelle Michèle Clément, réactualise d'une part, la figure de Scève à la Renaissance française, l'appuyant d'autre part, sur de nouvelles interprétations fondées toujours sur des discussions pertinentes qui relèvent de données originales et inédites.

2 Le texte, établi sur l'un des deux exemplaires conservés à la Bibliothèque Municipale de Lyon (Microcosme, Lyon, Jean de Tournes, 1562, cote: Rés. 355 902), est accompagné d'un apparat de notes qui témoignent d'une recherche approfondie autour de l'auteur et des questions érudites qui le concernent, mais aussi d'une réflexion critique constante qui permet de comprendre cette œuvre grâce aux annotations méticuleuses qui veulent de même accompagner et introduire le destinataire du texte à la complexe compréhension de l'ouvrage. Or, cette démarche scientifique rigoureuse n'est que la deuxième des 
étapes offertes par Michèle Clément - la troisième est construite sur un glossaire très riche (pp. 320-352) et sur une annexe au glossaire (p. 353) - puisque la première est entièrement occupée par une introduction de 129 pages où notre éditeur ouvre constamment ses argumentations au dialogue avec les critiques et les enquêtes qui précèdent son édition, apportant à la fois une lecture stimulante et nouvelle par son originalité. Partant de la tentative de dire comment on pourrait considérer ou définir la poésie de Microcosme, Michèle Clément se pose et propose en même temps au lecteur la question suivante: "[cette poésie] est-ce une poésie scientifique, une poésie encyclopédique ou une poésie philosophique?» (p. 14) et elle essaie de formuler une réponse prudente faisant appel aux études de A.-M. Schmidt, D. B. Wilson, M. Fumaroli, F. Hallyn, V.-L. Saulnier etc. Après avoir tenté une définition/distinction, dans une perspective historiographique, des catégories de poésie scientifique et de poésie philosophique, Michèle Clément souligne que «dans ce rapport à la science, Scève nous offre bien une poésie philosophique. Acceptons alors l'emploi de cette catégorie - dit l'A. L'on retrouve alors la définition que donne Ronsard de la philosophie et l'entreprise de mise en mots synthétique du monde. [...]. Du reste, affirme encore Michèle Clément «[...] ce n'est pas le traitement de la science dans un but didactique qui intéresse Scève et le poème n'a pas comme premier objectif de dire, ordonner ou transmettre le savoir scientifique [puisque], avec Microcosme, Scève fait de la poésie le lieu du savoir pensé contre la mort» (pp. 17-18). Donc, pourrait-on dire, savoir scientifique et discours philosophique s'entrelacent dans un tissu culturel dont les sources majeures, la Bible, la Margarita philosophica de Gregor Reisch, mais aussi «des sources supplémentaires, dont Pline, Bérose ou Politien» (p. 26) se mêlent à l'influence de Macrobe et de Martianus Capella, pour donner lieu à l'élaboration d'un ouvrage à propos duquel «il s'agit bien sûr de chercher, autant que possible et en toute conscience, du caractère fragile de la reconstruction, à retrouver les structures mentales dans lesquelles a travaillé Scève, les textes qu'il a lus pour les transformer en vers français, de chercher une intention (qui saurait s'en priver?) mais aussi et surtout, il s'agit de chercher le sens de ce texte pour un lecteur d'aujourd'hui et - c'est quasiment la même chose, ou son envers - chercher ce que ce texte dit qui n'est plus que difficilement audible aujourd'hui» (pp. 31-32). C'est ainsi que l'A. envisage un problème consubstantiel à Microcosme, qui est celui de l'herméneutique historique érudite, problème lié strictement, pour Michèle Clément, à la tentative de comprendre la raison pour laquelle Scève essaie de reconstituer en quelque sorte l'histoire originelle de l'humanité par le biais de l'idée de microcosme. Voilà donc le but final et constitutif de cette édition critique, par laquelle l'éditeur réfléchit aussi sur la proximité de Scève et de son Microcosme avec les Dialogues d'amour de Léon Hébreu, l'Historia ecclesiastica de Pierre Comestor (Pierre le Mangeur) et la Margarita philosophica de Gregor Reisch citée précédemment. Mais il ne s'agit pas seulement de cela, puisque Michèle Clément prolonge son enquête et ses réflexions par une tentative qui, nous semble-t-il, porte à des conclusions partageables. En effet, elle délie la notion ou catégorie de microcosme scévienne de celle de macrocosme, formulant des hypothèses et par la suite des argumentations convaincantes sur «la nécessité de se défaire d'une mauvaise dichotomie entre dignité et misère de l'homme. [...] Ce qui intéresse Scève, c'est la conjonction de la dignité et de la misère et la réversibilité permanente de l'une en l'autre, en particulier via la "vexation qui donne l'entendement"» (p. 42). Par cette ouverture dialogique qui concerne aussi le bien et le mal, notre éditeur dresse un discours tout à fait pertinent et intéressant sur la portée théologique de Microcosme, poème biblique, dit Michèle Clément, "dans son début», 
«encyclopédique sur sa fin» (pp. 44-45), mettant au centre du discours des observations critiques sur la notion catholique de felix culpa chez Scève au sein de son ouvrage. Mais par la reconstruction de la réception de la Bible dans Microcosme, Michèle Clément interroge la pratique ou, mieux encore, les pratiques poétiques de Scève, à partir du 'modèle' biblique, relevant que la technique la plus utilisée dans cet ouvrage est celle de l'amplification («[...] l'épisode de Caïn de même que celui de Babel sont hypertrophiés par Scève, qui ajoute à la Genèse des épisodes empruntés à Flavius Josèphe comme l'écroulement de la Tour de Babel ou inventés comme la combustion des offrandes d'Abel et de Caïn; quant aux interpolations, elles sont nombreuses et essentielles, véritables marques scéviennes, parfois en écho avec Délie et avec les blasons: beauté du corps d'Ève lors de sa création (I, 172-222), scène d'amour charnel entre Adam et Ève (I, 468-476), intelligence d'Ève (III, 177-209)», p. 52). La problématique de la réception de la Bible se lie encore aux questions concernant l'appartenance confessionnelle de Scève, si bien qu'elle donne à notre éditeur la matière nécessaire pour mettre en discussion les recherches sur ce sujet précédant ce travail, et pour discuter en même temps des différentes conjectures concernant cette appartenance, tiraillée, par les spécialistes, entre catholicisme, évangélisme ou encore entre protestantisme calvinien et protestantisme luthérien. Toutefois, par une démarche dans une perspective historiographique rigoureuse, Michèle clément conclut que les «cinq données théologiques (bonnes œuvres, felix culpa, libre arbitre, limbes et syndérèse) font de Microcosme, sans aucun doute, un poème catholique» (p. 87).

Nous n'avons donc qu'à saluer ce travail important et rigoureux qui permet de mieux comprendre un auteur de l'envergure de Scève, auteur très complexe qui pourra aussi être l'objet, par l'intermédiaire de cette belle édition, d'une relecture à proposer aux spécialistes du secteur et d'une première réflexion à offrir aux étudiants et aux doctorants seiziémistes. 